\title{
Representación social del fracaso empresarial de las PYME de Bogotá a partir de las investigaciones realizadas entre 2007 y el 2017
}

\section{Social representation of the business failure of SME in Bogota from investiga- tions conducted in 2007-2017 period}

\author{
Claudia Patricia Gómez Espinosa ${ }^{1}$ \\ Sandra Cristina Castro Becerra ${ }^{2}$ \\ Luisa Alejandra Rojas Melo ${ }^{3}$ \\ DOI: 10.29151/hojasyhablas.n18a2
}

\section{Resumen}

El artículo identifica aspectos de la representación social del fracaso empresarial de las pequeñas y medianas empresas (PYME) de Bogotá, a partir de resultados de investigaciones publicadas en revistas académicas entre 2000 y 2017, como parte del proyecto "Elementos culturales presentes en la representación social del fracaso empresarial de las PYME de Bogotá", financiado y desarrollado por la Universidad Cooperativa de Colombia, y cuyo enfoque es de tipo cualitativo. El proceso metodológico corresponde al análisis de contenido temático y se enfoca en identificar los núcleos de sentido al interior de las categorías y variables internas de la empresa. Para determinar la muestra, se revisaron 100 artículos y se seleccionaron 30. Dentro de la modelación se tomó en cuenta el grado de incidencia que los autores dan al fracaso empresarial, determinando el núcleo y la periferia de la representación. Una vez establecido el sistema de relación, se finalizó con un análisis hermenéutico, donde se evidencia que las investigaciones están centradas en gestión y finanzas (núcleo) y en mercadeo e innovación (periferia).

Palabras claves: PYME; fracaso empresarial; representación social; gestión empresarial; finanzas.

\section{Abstract}

This article identifies relevant aspects related with social representation about business failure of SME in Bogotá, throughout investigations published between 2000 and 2017, as a part of "Cultural issues in social representation of business failure of SME of Bogota" Project, which was developed by Universidad Cooperativa de Colombia. The methodology uses a qualitative type, doing a content analysis and identifying the cores of sense into microenvironment. In order to determine the sample, 100 academic paper were revised and 30 were selected. The variable to systematize information was business failure, analyzing the incidence of finances, logistic, marketing and innovation.

Keywords: SME; business failure; social representation; business management; finances.

\footnotetext{
${ }^{1}$ Magíster en Psicología del Consumidor. Docente Universidad Cooperativa de Colombia. E-mail: claudiap.gomeze@campusucc.edu.co ORCID: 0000-00028691-4799

${ }^{2}$ Magíster en Psicología del Consumidor. Docente Universidad Cooperativa de Colombia. E-mail: sandra.castro@campusucc.edu.co ORCID: 0000-00018889-9687

${ }^{3}$ Magíster en Investigación Social Interdisciplinaria. Docente Universidad Cooperativa de Colombia. E-mail: luisa.rojas@campusucc.edu.co ORCID: 00000002-7371-1319
} 


\section{Introducción}

De manera constante, en nuestro país se generan proyectos de creación de empresas, en su mayoría pequeñas y medianas (en adelante PYME), las cuales constituyen una oportunidad para mejorar el nivel de vida, mediante la producción y distribución de bienes y servicios. El papel de estas empresas como generadoras de empleo o alternativas para la producción es reconocido en diferentes estudios. En el 2011, Puyana afirmaba que "las PYME son el motor de la economía, generan más del 50\% del empleo nacional; significan el 36\% del valor agregado industrial, el $92 \%$ de los establecimientos comerciales y el $40 \%$ de la producción del país" (p. 1). Para el año 2016, dicha situación no había cambiado, pues según las estadísticas del Departamento Nacional de Estadística - Dane (2016b), las PYME soportan alrededor del 67\% del empleo y aportan el 28\% al Producto Interno Bruto (PIB), haciéndolas indispensables para el sistema productivo colombiano.

Según las estadísticas de Confecámaras, en el año 2016 se crearon en Colombia 299.632 unidades productivas, de las cuales 76.794 son sociedades y 222.838 personas naturales. La mayor concentración de las PYME se ubica en Bogotá, Cundinamarca, Atlántico, Antioquia, Valle del Cauca y Santander, para un total del 66\% del aparato productivo nacional (ANIF, 2016, p. 60). En el caso de Bogotá, las PYME aportan el 24.95\% al PIB colombiano, y la ciudad acoge a 740.069 PYME, correspondientes al 29,38\% del total nacional (Dane, 2016a).

Pese a su importancia, un gran número de las PYME no logra perdurar en el mercado. De acuerdo con Velasco (2008) "un número considerable de PYME no logra establecerse; de las que sobreviven, el 30\% no supera el primer año de vida y se considera que después de 10 años desaparece en su tamaño el 70\% de las que aún existe" (p. 20). Una década después, la situación descrita no ha cambiado significativamente. Según el estudio realizado por la Dirección de Gestión del Conocimiento de la Cámara de Comercio de Bogotá (2019) “cerca de 18.752 empresas se cierran cada año en Bogotá, por lo cual se disminuye la capacidad productiva de la ciudad, se reducen los ingresos tanto públicos como privados, se pierden esfuerzos y experiencia de los emprendedores y se limitan las fuentes de empleo" (p. 2). Por ello, la pregunta sobre las causas de la situación de las PYME sigue siendo válida y el problema de investigación sigue vigente.

En el país existen numerosos estudios que analizan la situación de las PYME. Los primeros se realizaron entre 1990 y el 2010, constituyendo un gran aporte a investigaciones posteriores, entre ellos se destaca el desarrollado por la Fundación para el Desarrollo Sostenible de las pequeñas y medianas empresas en América Latina (FUNDES, 2003), denominado La realidad de la PYME colombiana. En él se plantea que los principales obstáculos para el desarrollo de las PYME eran el acceso al financiamiento, la calidad y disponibilidad del recurso humano, el acceso a mercados externos y la legislación laboral. A partir de allí, se diseñaron marcos jurídicos, como la Ley Pyme, se plantearon programas de desarrollo estatales y gremiales, así como se desarrollaron diferentes propuestas de formación y fortalecimiento a instituciones como la Cámara de Comercio y las Universidades.

A pesar de lo anterior, la problemática sobre la baja permanencia en el mercado sigue estando presente. Por esto, cabe preguntarse, ¿qué se plantean los investigadores en los estudios recientes?, ya que, de acuerdo con Méndez (2009) 
en su libro Tecnologías y herramientas de gestión. Caso grandes, medianas y pequeñas empresas en Bogotá, "en esta primera década del siglo XXI se siguen cometiendo los mismos errores de los años ochenta y noventa en la intención de aplicar tecnologías o herramientas de gestión" ( $p$. 46). De ahí la importancia de analizar la situación desde otras perspectivas.

Por ello, es necesario indagar sobre los aspectos que ya se han investigado, ya que esto permitiría encontrar otra perspectiva de trabajo que redunde en el fortalecimiento de dichas empresas. De ahí, la importancia de revisar la literatura científica publicada, analizando la forma en que se planteó el problema, las categorías o variables incluidas en los estudios y sus resultados. Tal es el caso del proyecto "Elementos culturales presentes en la representación social del fracaso empresarial de las PYME de Bogotá", el cual indaga sobre el fracaso empresarial desde la teoría de las representaciones, tomando en cuenta tres aspectos: las investigaciones sobre las PYME, la historia de vida de los empresarios y la experiencia de personas que han trabajado en instituciones de apoyo. Este artículo presenta los resultados del primer aspecto.

Para la investigación se tomó como enfoque teórico las representaciones sociales (RS), el cual parte de la existencia de modelos mentales previos a las acciones (Ricoeur 2000; Ibáñez 2001), y que dichos paradigmas están enlazados a la cultura, siendo elementos que influyen en la toma de decisiones, en este caso las de los investigadores. En este sentido, tanto la elección del tema, como la inclusión y la exclusión de criterios o variables son analizados como parte del discurso cultural de los mismos investigadores, el cual, de acuerdo con la teoría de las RS, influye en el desarrollo y los resultados de las investigaciones.
Ahora bien, la cultura es un término polisémico que puede ser analizado desde diferentes enfoques; sin embargo, se puede entender como un elemento cohesionador de los grupos humanos que permite su identificación y agremiación, $y$, que, en muchos aspectos, determina la forma de actuación individual. En este sentido, puede entenderse como "la programación colectiva de la mente que distingue los miembros de un grupo" (Hofstede Citado por Páramo 2000. p. 6). En términos de Vargas (2007) la cultura es como un conjunto de ideas compartidas por miembros de un grupo, o, en palabras de Murdock (1945) son las normas, creencias y actitudes con las cuales los individuos actúan. De lo anterior, se puede inferir que una forma en la que se puede comprender dicha programación es a través de los sistemas ideacionales, entre ellos, las representaciones sociales.

En palabras de Jodelet (1988 citado por Vecina, 2009), al ser las representaciones sociales una forma de conocimiento social, como la actividad mental de los individuos y grupos, utilizada para mantener una posición frente a determinados acontecimientos $\mathrm{u}$ objetos, inciden en nuestra forma de pensar, de interpretar las acciones". De ahí que, la representación social del fracaso empresarial que tienen los investigadores influye en la conceptualización del problema y las soluciones propuestas. Dichas ideas se pueden analizar desde los textos que se han producido al respecto.

En este sentido, los artículos publicados sobre el fracaso de las PYME son la entrada para comprender los sistemas ideacionales de los investigadores, ya que, de acuerdo con Ricoeur (2000) el texto es un discurso fijado por medio de la escritura. Por ello, estos textos permiten analizar los sistemas ideacionales de los autores. 
Así, a través de la lectura analítica de los artículos se pueden evidenciar las relaciones que como grupo tienen los que se acercan al tema, lo cual, finalmente conduce a una mirada sobre los ejes conceptuales y culturales presentes en ellos, pues las investigaciones están mediadas por las ideas que unos y otros tienen sobre los objetos o los fenómenos. En este sentido, los sistemas ideacionales son parte constitutiva de la práctica investigativa. Ahora bien,

$\mathrm{Al}$ aludir a los sistemas ideacionales se quiere señalar que estos constructos no son simplemente sistemas de ideas que operan sólo en función de abstracciones que existen en estado aislado, sino constructos que permiten objetivar de manera simultánea los efectos de las condiciones materiales con los procesos relativamente autónomos de las elaboraciones simbólicas (Serna, Oviedo y Bravo, 2010, p. 43).

Así, las ideas pueden ser consideradas como guías, modelos, paradigmas o puntos desde los cuales se comprende, explica e interpreta la realidad y la experiencia. Dichas selecciones se relacionan unas con otras conformando sistemas ideacionales. De acuerdo con Absalón Jiménez (2012) "el dispositivo imaginario, las imágenes y los símbolos (...) forman parte de complejos y compuestos sistemas, asegurando a un grupo social un esquema colectivo de interpretación y de experiencia” (p. 179).

Para Rodríguez, García y Jodelet (2007), las representaciones sociales están constituidas por un contenido o la información es precisa por un cumulo de saberes, conocimientos que se extraen de los grupos, las comunidades, los colectivos sobre el evento, hecho o fenómeno social existente. De igual modo, para Florez Ospina (2009) la teoría de las representaciones sociales brinda un abanico de posibilidades para explicar los comportamientos de las personas, y permite justificar su realidad a través de la cultura y las estructuras sociales. En esto concuerda con Arbic (2001), quien afirma que las representaciones sociales funcionan como un sistema de interpretación de la realidad que rige las relaciones entre los actores sociales tanto en su ambiente físico como en el social. Por lo anterior, la forma en que se comprende o interpreta al fenómeno del fracaso empresarial de las PYME está mediado por un sistema de ideas, el cual puede ser entendido a partir de la figuración de las RS.

Para Ibáñez (2001), la RS es una unidad funcional de elementos heterogéneos fuertemente organizada y que funciona como una estructura organizadora. La estructura figurativa se construye a partir de un concepto central, al cual se le denomina núcleo, y una serie de conceptos o imágenes que están en la periferia y se relacionan en dos sentidos: 1) con el núcleo, y 2) entre ellos. Los dos aspectos constituyen un conjunto. Retomando a Moscovici, este mismo autor señala que existen tres ejes en torno a los cuales se estructuran los componentes de una representación: actitud, información y campo de representación. La actitud tiene que ver con la disposición de las personas frente al objeto de la representación. La segunda (información) está determinada por las condiciones de acceso del grupo. Finalmente, el campo de representación, el cual comprende la figuración o articulación de los elementos alrededor de un núcleo que se construye a partir del proceso de objetivación.

En el núcleo se organizan y jerarquizan los diversos contenidos conceptuales, los cuales se transforman en imágenes o elementos figurativos. El núcleo está integrado por los elementos centrales, aquellos que se constituyen como los 
predominantes a partir del consenso social (anclaje). La periferia, por aquellos que, aunque presentes, son minoritarios o distantes del centro (Ibáñez, 2001). Lo anterior, permite visualizar y analizar la representación y las articulaciones entre los conceptos que la integran, mostrando así, los enfoques, las tendencias y las creencias que tienen los investigadores frente al problema del fracaso empresarial de las PYME de Bogotá.

Conforme a lo expuesto, el núcleo de la RS está conformado por los aspectos predominantemente analizados dentro de las investigaciones, es decir, las categorías o variables que frecuentemente son adoptadas por los investigadores, las cuales constituyen los elementos anclados a la representación del problema. La periferia por los elementos que, aunque son tenidos en cuenta, han sido poco explorados o se consideran menos relevantes.

\section{Metodología}

Esta es una investigación cualitativa, en la que se busca modelar la representación social del fracaso empresarial a partir de los resultados de investigación sobre el tema que han sido publicados en revistas científicas en los últimos diez años. Para comenzar, se realizó una búsqueda de artículos en diferentes bases de datos como: Science Direct, ISI y Publindex que trabajaran el problema de la permanencia en el mercado de las PYME. En dicha indagación se recopilaron 100 artículos sobre el tema. A dichos documentos se les realizó un proceso de selección a partir de los siguientes criterios: publicaciones de los últimos 10 años (2007 - 2017); que indagaran sobre el fracaso empresarial de las PYME; que correspondieran a Bogotá o Colombia; que tuvieran un ejercicio de campo, es decir, que presentaran un diseño en el que se indagara directamente en y con las PYME.
En este sentido, se excluyen los textos que analizaban situaciones particulares de otras regiones, estudios enfocados a la revisión teórica del problema, artículos que compararan a Colombia con otros países y los que analizaban el fracaso empresarial de las micros o de las grandes empresas, y aquellos que analizaban el problema a partir de otros artículos (fuentes secundarias). Una vez realizada la selección se determinó que 30 de ellos cumplían con los criterios de selección, por lo que fueron incluidos en la muestra.

Una vez seleccionado el corpus, se procedió a hacer un análisis de contenido, entendido como un conjunto de técnicas o pasos que tienden a explicar y sistematizar la información de los mensajes comunicativos, en este sentido, de los artículos científicos objeto del presente estudio. Por ello, "Consiste básicamente en la descomposición, fragmentación del texto en unidades constitutivas para su posterior codificación según un sistema de categorías, generalmente preestablecido. Una vez fragmentado y codificado el texto, se aplican diferentes técnicas de análisis" (García citado en Toro, 2010, p. 382).

Para la realización de dicho análisis, se desarrollaron las siguientes etapas: 1) Determinar los objetos o temas de análisis; 2) Establecer las reglas de codificación; 3) Organizar el sistema de categorías; 4) Comprobar la fiabilidad del sistema de codificación y categorización.

Para determinar los objetos y temas de análisis, inicialmente se realizó una indagación sobre la definición del fracaso empresarial que permitiera articular los elementos centrales de indagación. Según Romero (2015), las definiciones de fracaso, a partir de diferentes autores se resumen a continuación: 


\section{Tabla 1. Definiciones de fracaso empresarial}

\begin{tabular}{|c|c|c|c|}
\hline Autor & País & Término & Definición \\
\hline Beaver. 1966 & $\begin{array}{l}\text { Estados } \\
\text { Unidos }\end{array}$ & Fracaso & $\begin{array}{l}\text { Dificultad para atender deudas (obligaciones } \\
\text { financieras) }\end{array}$ \\
\hline Altman. 1968 & Espafia & Quiebra & Catalogadas legalmente en quiebra \\
\hline $\begin{array}{l}\text { Correa, Acosta } \\
\text { Gonzâlez, } 2003\end{array}$ & \& Espafia & Quiebra & Patrimonio negativo o quiebra técnica \\
\hline $\begin{array}{l}\text { Somoza } \\
\text { Vallverdú, } 2003\end{array}$ & \& Espania & Fracaso & $\begin{array}{l}\text { Suspensión de pagos, insolvencia financiera } \\
\text { declarada. Ley Concursal } 22 / 2003\end{array}$ \\
\hline Martinez, 2003 & Colombia & Fragilidad & $\begin{array}{l}\text { La empresa ingresó en un acuerdo de } \\
\text { reestructuración de pagos o liquidación } \\
\text { obligatoria }\end{array}$ \\
\hline Platt \& Platt, 200 & $\begin{array}{l}04 \text { Estados } \\
\text { Unidos }\end{array}$ & Fracaso & Fracaso financiero \\
\hline $\begin{array}{l}\text { Calvo, Garcia } \\
\text { Madrid, } 2006\end{array}$ & \& Colombia & Fracaso & Riesgo financiero alto \\
\hline $\begin{array}{l}\text { Acosta } \\
\text { Fernández, } 2007\end{array}$ & \& España & Fracaso & Quiebra empresarial \\
\hline $\begin{array}{l}\text { Gómez, Torre } \\
\text { Román, } 2008\end{array}$ & \& España & Fracaso financiero & $\begin{array}{l}\text { Suspensión de pagos o quiebra legal de acuerdo } \\
\text { con la Ley Concursal Espaniola }\end{array}$ \\
\hline Rubio Misas, 200 & 08 Españia & Quiebra & Patrimonio negativo o quiebra técnica \\
\hline $\begin{array}{l}\text { Mora \& Gonzále } \\
2009\end{array}$ & ez, España & Fracaso & $\begin{array}{l}\text { Liquidación voluntaria, liquidación obligatoria, } \\
\text { acuerdo de reestructuración o concordato }\end{array}$ \\
\hline $\begin{array}{l}\text { Labatut, Pozue } \\
\& \text { Veres, } 2009\end{array}$ & elo España & Fracaso & $\begin{array}{l}\text { Catalogado legalmente en quiebra. Ley } \\
\text { Concursal } 22 / 2003\end{array}$ \\
\hline $\begin{array}{l}\text { Arquero, Abad } \\
\text { Jiménez, } 2009\end{array}$ & \& España & Fracaso financiero & $\begin{array}{l}\text { Cuando el deudor es incapaz de atender a sus } \\
\text { compromisos }\end{array}$ \\
\hline
\end{tabular}




$\begin{array}{lll}\begin{array}{l}\text { Veres, Labatut \& Espafia } \\ \text { Pozuelo, 2010 }\end{array} & \text { Fracaso } & \begin{array}{l}\text { Calificaciones juridicas de suspensión de pagos } \\ \text { y quiebra. Ley Concursal } 22 / 2003\end{array} \\ \begin{array}{l}\text { Pozuelo, Labatut España } \\ \text { \& Veres, 2010 }\end{array} & \text { Fracaso } & \begin{array}{l}\text { Calificaciones juridicas de suspensión de pagos } \\ \text { y quiebra }\end{array} \\ \text { Manzaneque, 2010 Espafia } & \text { Fracaso } & \text { Suspensión de pagos o quiebra }\end{array}$

Banegas \& Garcia, 2010

La existencia de resultados de explotación y/o patrimonio neto negativo durante tres ejercicios consecutivos

Llano, Pifieiro \&Espafia Rodriguez, 2011

Camacho, Salazar Colombia \& León, 2013

Pérez, González \& Colombia Fragilidad Lopera, 2013

Garcia \& Mures, Espafia Fracaso 2013

$\begin{array}{ll}\text { Caro, Diaz \& Argentina } & \begin{array}{l}\text { Quiebra/crisis } \\ \text { financiera }\end{array}\end{array}$

Fracaso financiero Situación concursal o liquidación lempresas fallidas

Quiebra o iliquidez Incapacidad de atender obligaciones financieras a su vencimiento. Suspensión de pagos o concurso de acreedores. Patrimonio neto contable negativo

Reorganización, concordato, reestructuración y liquidación

Suspensión de pagos, quiebra y concurso de acreedores de acuerdo con la Ley Concursal espafiola

En cesación de pagos con concurso preventivo o pérdidas que absorben sus ganancias o capital

Fuente: Romero, 2015

De acuerdo con lo anterior, el fracaso empresarial se ha enmarcado principalmente en definiciones jurídicas y financieras, entonces el fracaso financiero o quiebra, corresponde a la escasez del flujo de caja, incumplimiento de pagos, entre otros que, afectan la gestión de la empresa, así como siempre se están buscando estrategias en ese orden, desconociendo otras que pueden generar ventaja competitiva y evitar dicho fracaso (Romero, 2013). En este sentido, Porter, Kramer y Lorsch (2009) define la cadena de valor como actividades propias que se relacionan entre si y que tienen como objetivo aportar el mayor valor posible para el cliente y como una herramienta estratégica de análisis para identificar las ventajas competitivas de un negocio frente al mercado.

Teniendo en cuenta esto, se planteó la necesidad de integrar todos los aspectos funcionales de la empresa, en este caso, los relacionados con las variables internas de la misma, de tal forma que permitiera hacer una agrupación de un campo de gran heterogeneidad, como lo es el de las categorías o variables em- 
pleadas por los diferentes investigadores. Para lograrlo, se buscó seleccionar categorías de carácter general de la Administración de Empresas y se pueden observar en la siguiente tabla.

\section{Tabla 2. Sistema de categorías}

\section{Criterio}

Dimensión

\begin{tabular}{|c|c|c|}
\hline \multirow{8}{*}{$\begin{array}{l}\text { Var } \\
\text { iables } \\
\text { internas de } \\
\text { la Empresa }\end{array}$} & \multirow[t]{4}{*}{ Financiero } & Acceso al Financiamiento \\
\hline & & Estructura de costos \\
\hline & & Manejo de la información financiera \\
\hline & & Flujo de caja \\
\hline & \multirow[t]{3}{*}{ Recurso humano } & Conocimiento y competencias \\
\hline & & Procesos de selección y contratación \\
\hline & & Relaciones interpersonales \\
\hline & \multirow[t]{3}{*}{ Innovación y tecnología } & Acceso \\
\hline & & Conocimiento \\
\hline & & Implementación \\
\hline & \multirow[t]{5}{*}{ Mercadeo } & Producto \\
\hline & & Precio \\
\hline & & Plaza \\
\hline & & Promoción \\
\hline & & Consumidor \\
\hline & \multirow[t]{4}{*}{ Gestión organizacional } & Planeación \\
\hline & & Organización \\
\hline & & Dirección \\
\hline & & Control \\
\hline
\end{tabular}

Fuente: Elaboración propia, 2019. 
Una vez establecidas las categorías, se plantearon las reglas de codificación a través del diseño de unas fichas de lectura analítica de los artículos con el fin de identificar las categorías o variables tenidas en cuenta en cada uno, ubicándolos en una de las categorías y dimensiones propuestas en la tabla 3. Posteriormente, a partir de los resultados de cada artículo, se analizó cuáles de dichas categorías fueron evaluadas por los autores como las determinantes del fracaso empresarial, cuáles de mediana incidencia y cuáles de baja incidencia.

A estos elementos se les asignó una calificación cualitativa, simbolizada de manera numérica de la siguiente forma: 5 para el elemento predominante en la representación, es decir el factor al que el autor considera la o las causas más importantes del fracaso de las PYME; 3, los elementos que se consideran secundarios o de mediana incidencia, y 1 para los que estando presentes se consideran de baja importancia en el contexto general. Cabe aclarar que el objetivo fue identificar la posición de los autores, y no la de quien analiza la información, por lo que dichos aspectos se categorizaron de acuerdo con las proposiciones usadas por ellos.

Para efecto de la representación social del fracaso, se promediaron las calificaciones del total de los artículos publicados sobre el fracaso empresarial en las diferentes categorías de las variables internas de la Empresa, cuyo promedio del grado de incidencia corresponde a las categorías establecidas en la Tabla 3.

Tabla 3. Grado de incidencia las categorías encontradas en el análisis de los artículos obtenidas en los listados variables de las variables internas de la Empresa

\begin{tabular}{lcc}
\hline Categoría & Incidencia & $\begin{array}{c}\text { Promedio \# } \\
\text { Art }\end{array}$ \\
\hline Financiero & 1.73 & 6 \\
Recurso Humano & 1.78 & 7 \\
Innovación y Tecnología & 1.44 & 4 \\
Mercadeo & 0.67 & 2 \\
Gestión Organizacional & 2.17 & 11 \\
Total & & 30 \\
\hline
\end{tabular}

Fuente: Elaboración propia, 2019. 
De esta manera, a cada categoría se le dio un peso dentro de la representación, no solo por el número de artículos que analizaron la categoría, sino por el grado de incidencia en el fenómeno que le fue dado por los autores. Así, las categorías que tenían presencia en un mayor número de artículos y que adicionalmente eran consideradas por los autores como las de mayor incidencia, consolidaron el núcleo de la representación; mientras que, aquellas que se estudiaron en menos artículos o que a pesar de ser tenidas en cuenta no fueron identificadas como de relevancia en el problema, constituyeron la periferia.

\section{Resultados y discusión}

Una vez analizada la información, se realizó la modelación gráfica de la representación, a partir del sistema de categorías presentado en la tabla 2.

\section{Gráfico 1. Organización de la Representación Social del Fracaso Empresarial desde las di- mensiones del Variables internas de la Empresa}

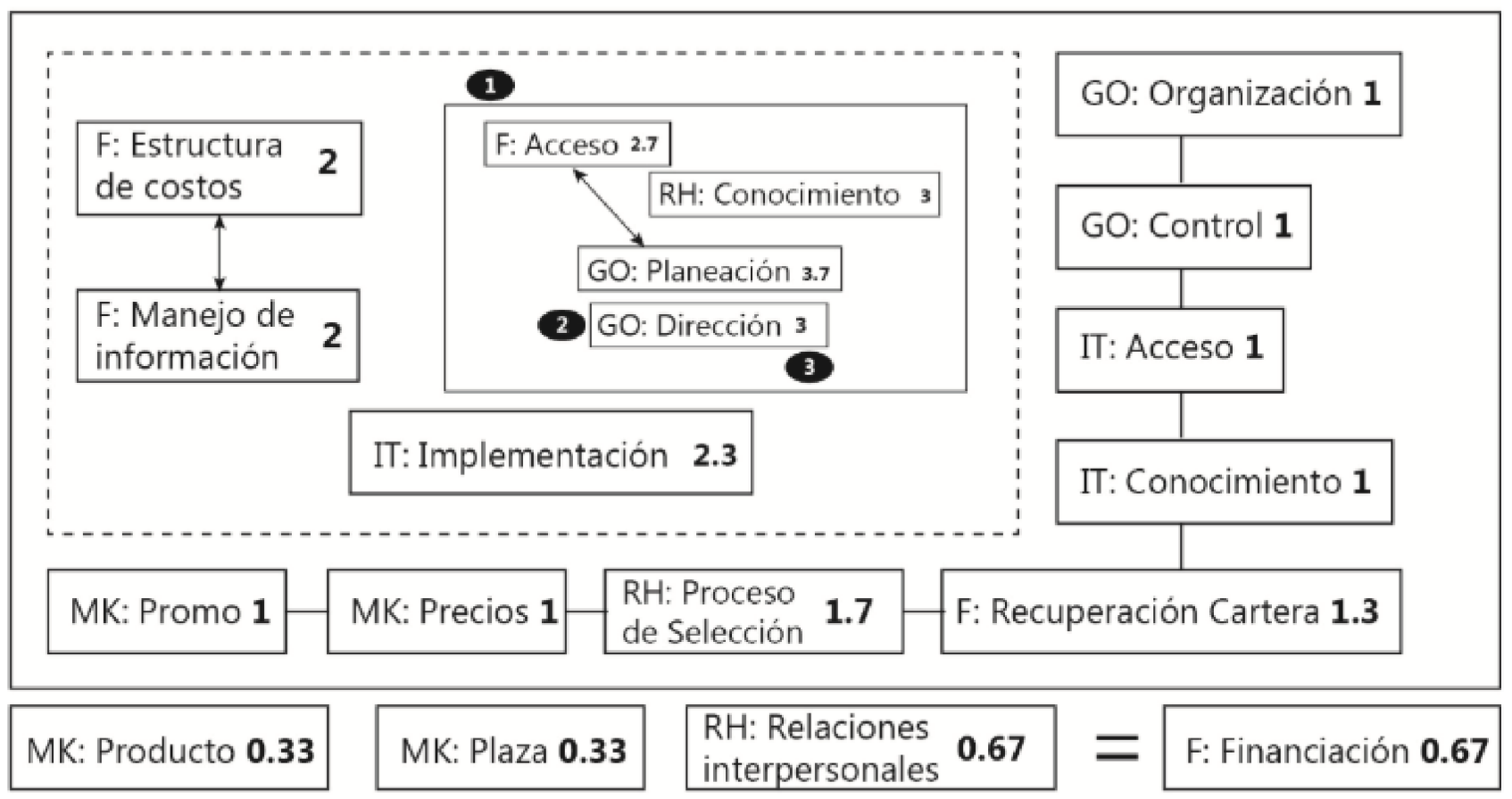

Fuente: Elaboración propia, 2019.

En el gráfico 1 se evidencia el interés de las y los investigadores en abordar el tema del fracaso empresarial desde diferentes miradas de las ciencias sociales, a saber, la Administración de Empresas, la Contaduría Pública y el Mercadeo, siendo la variable financiera aquella con mayor interés; constituyendo uno de los aspectos centrales para ubicarse en el núcleo de la representación. El otro aspecto predominante está relacionado con la gestión organizacional, especialmente en la dimensión denominada planeación.
La organización de la RS del fracaso empresarial desde el criterio del micro-entorno, muestra que existe un buen número de estudios acerca de las dimensiones de las categorías financiera (F), recurso humano $(\mathrm{RH})$, innovación y tecnología (IT), mercadeo (MK) y gestión organizacional (GO), donde el mayor grado de incidencia y recurrencia se ve reflejado en el núcleo, como lo son: F: Acceso, RH: Conocimiento, GO: Planeación y GO: Dirección, en los artículos revisados. Estas también constituyen el núcleo. Otras dimensiones 
que se tienen en cuenta en los estudios, pero que son menos recurrentes o consideradas como de mediana incidencia por los investigadores son: $\mathrm{F}$. Estructura de Costos, F: manejo de información, IT: Implementación. Las demás dimensiones se encuentran ubicadas en la periferia de la organización de la representación social del fracaso empresarial, desde las dimensiones del micro-entorno. Dichas dimensiones son aquellas en las que, aunque existen estudios al respecto, han sido poco trabajadas en los estudios sobre la baja permanencia de las PYME en Bogotá o son consideradas por los autores como de baja incidencia.

Adicionalmente, en el análisis de la información se determinó que en los estudios de fracaso empresarial es de gran interés para los investigadores indagar el fenómeno de la gestión financiera y la gestión organizacional, de los cuales existe una relación de doble vía entre las fuentes de acceso de financiación y el proceso de planeación; entendiéndose el acceso a la Financiación, como el conjunto de herramientas empleadas por las distintas instituciones financieras para realizar la evaluación de las solicitudes de crédito (Chorro, 2010). Lo anterior, obedece a la necesidad de identificar las causas y los síntomas de una empresa que fracasa a partir de variables, en donde el fracaso empresarial se deriva de la financiación por problemas estrictamente financieros.

Por lo tanto, los resultados principales muestran que las variables financieras determinantes del fracaso empresarial para la pequeña y mediana empresa en el país son: Pasivo no corriente / Activo total, Pasivo total (Activo corriente-Inventario) /Activo total. En palabras de Fredy Romero (2016) "La pequeña y la mediana empresa que fracasa se caracteriza por tener un mayor grado de apalancamiento de largo plazo, un pasivo total alto, una baja concentración de activos líquidos con relación a su activo total, los cuales no alcanzan a cubrir los pagos de la deuda" (p. 273).
Estas variables financieras ponen de manifiesto que los investigadores consideran que la pequeña y la mediana empresa que fracasa se caracteriza por tener un mayor grado de apalancamiento de largo plazo, un pasivo total alto, una baja concentración de activos líquidos con relación a su activo total, los cuales no alcanzan a cubrir los pagos de la deuda. Por tanto, sus principales problemas son de liquidez y endeudamiento. (Romero, 2013).

De otra parte, en el núcleo, pero con una menor incidencia se ubican: la estructura de costos y el manejo de la información financiera, pues en los estudios analizados se evidencia que las PYME fracasadas tenían problemas de solvencia y capacidad de pago, lo cual redunda en la disminución del capital de trabajo. En palabras de Romero, Melgarejo y Vera (2015) "por cada peso de obligación vigente de corto plazo, las empresas sanas contaban con 1.90 pesos para respaldarlos en 2009 y 1.62 en 2013, mientras que las empresas fracasadas contaban con 1.16 en 2009 y tan solo 0.99 pesos en 2013 , lo que hace evidente las menores solvencia y capacidad de pago de estas últimas; sin contar que la prueba [ha arrojado] una dependencia directa de estas empresas de sus inventarios, al no poder atender las obligaciones corrientes sin necesidad de venderlos, además de que el disponible constituye en promedio solo el $2.39 \%$, frente al $9.73 \%$ en las empresas sanas" (p. $38)$.

La segunda variable que se relaciona en el núcleo, con respecto al fracaso empresarial, es la gestión empresarial. Hay un interés de los investigadores por abordarla desde el proceso administrativo en la planeación, definida como la fijación de los objetivos, estrategias, políticas, programas, procedimientos y presupuestos, partiendo de una previsión, para que el organismo social cuente con las bases requeridas para encausar correctamente las otras fases del proceso administrativo (Luna, 2014). 
El estudio realizado por Pardo y Alfonso (2015) demostró que las principales variables están relacionadas con la planeación, lo cual se traduce en carencia de indicadores de gestión, por ende la administración como herramienta indica que es necesario planificar en función a objetivos claramente definidos, en atención a un norte alcanzable, con estrategias dinámicas y con flexibilidad, puesto que la flexibilidad permite, en el cambiante mundo de hoy, identificar riesgos que al inicio la organización no se había planteado. Lo anterior, permite inferir que el éxito de un emprendimiento es clave la experiencia y las herramientas gerenciales.

Además, los empresarios enfocan sus esfuerzos en la gestión del día a día, sin tener en cuenta el establecimiento de un plan a futuro que constituya el plan de acción de la empresa (Beltran, 2006). Actualmente, se afirma que la planeación estratégica adopta patrones de comportamiento de acuerdo con el ciclo de desarrollo de la empresa: va de un ciclo de emprendedor informal a uno de planeación formal, lo que afecta directamente la identidad de la empresa (Castaño, 2011).

De igual manera, Chaparro (2012) argumenta que, en la actualidad, la administración de muchas PYME, reconocen que los conocimientos y la institución por sí solos no son suficientes para guiar el destino de sus organizaciones, ya que el entorno y el ambiente cambian; por ello se recurre cada vez más al uso de la planeación estratégica, para poder planear y controlar su crecimiento, buscando aumentar sus utilidades y reducir los riesgos para lograr la supervivencia de la empresa en el mercado.

En segundo lugar, según los análisis de la información encontrada, en el núcleo, pero con menos incidencia, se evidencia la implementación de la innovación, seguido del recurso humano desde sus competencias. Como primera instancia la innovación es el arte de convertir las ideas y el conocimiento en productos, procesos o servicios nuevos o mejorados que el mercado reconozca y valore (Vásquez, Escudero y Gabín, 2010). Por ello, se puede inferir que, para los teóricos la implementación de la innovación va da la mano con el bajo nivel de conocimiento y competencias del recurso humano. Siendo estos elementos una segunda relación interna dentro del núcleo de la representación.

Conforme las organizaciones van creciendo, estas deben estar a la vanguardia del desarrollo de innovaciones para la satisfacción de los mercados. Por tanto, la innovación de producto es un proceso intensivo en conocimiento de tecnología, de mercado y de la organización interna de los recursos técnicos, económicos y humanos (Suarez, De la Rosa, Jimenes y Toyos, 2009). De otra parte, la innovación de los servicios se ve reflejada en las nuevas tecnologías, especialmente en la digitalización y movilidad, afectando a los consumidores y a las empresas y generando que la información se obtenga de manera inmediata. Por ende, las organizaciones se ven enfrentadas a estos nuevos retos, a fin de no quedarse en las dinámicas tradicionales que conduce al fracaso empresarial (Villaseca, 2014).

Finalmente, en la innovación de los procesos, según Berumen (2008), el problema se agudiza cuando debido a cuestiones relacionadas con los drásticos cambios en la agenda o en la estrategia, el interés por los procesos de innovación se hace más lento en su implementación, con tendencia a desaparecer. Por tanto, es importante que las organizaciones dediquen esfuerzos a procesos científicos, tecnológicos, organizacionales, financieros y comerciales. Entonces, también los investigadores dan cuenta de que las TIC hacen parte fundamental de los procesos de la organización. Sin embargo, estos problemas son considerados de menor incidencia frente a lo financiero y a las dificultades de planeación y dirección, por lo que constituyen elementos que empiezan a formar parte de la periferia de la representación social. 
Conforme a los estudios, se encontró que el recurso humano también hace parte del núcleo como información relevante en la investigación desde el conocimiento y las habilidades. Por ende, según Chiavenato (2009), administrar a las personas y las competencias humanas representa hoy una cuestión estratégica para las empresas. Se trata de una actividad de suma importancia para quedar limitada a un departamento de la empresa, pues ya no es posible que solamente un área de la empresa consiga centralizar y encerrar en sus manos la administración de las competencias y los talentos de todas las personas de la organización.

Ahora, en pleno auge de la cuarta revolución industrial, en la cual el cambio, la competitividad, la imprevisibilidad y la incertidumbre, constituyen los desafíos básicos de la empresa, la gestión del talento humano se debe desarrollar desde la innovación, teniendo en cuenta que es el motor de funcionamiento de una organización para el cumplimento de los objetivos estratégicos, porque las personas hacen parte fundamental de la toma de decisiones.

Es así como los teóricos están indagando estas variables, para identificar si tienen incidencia o no en el fracaso empresarial. Al respecto, la investigación realizada por Calderon y Castaño (2005), sobre la Administración en América Latina, muestra que la transformación experimentada por la función de personal ha contribuido a que la dirección de la organización haya empezado a considerar la gestión de lo humano y lo cultural en sus planteamientos estratégicos, lo que ha permitido que los académicos se interesen por investigar en este campo de la administración como parte primordial del progreso y desempeño de las organizaciones. Según estas investigaciones, se identifica el interés por las competencias mínimas que deben tener los líderes de las organizaciones, las potencialidades existentes para generar capital humano, la necesidad de consolidar o construir sus sistemas de valores y proveer un ambiente laboral acordes con las necesidades de estas, con el fin de permanecer en el mercado actual (Calderon y Castaño, 2005).

En la periferia, según el análisis realizado, se ubica el proceso de organización como una variable que no es objeto de estudio para los teóricos, sin embargo, desde la literatura el proceso organizacional hace parte fundamental para cumplir los objetivos estratégicos de la organización (Dichiara, 2011). En la era actual, lo que hace sostenerse en el futuro a las organizaciones es ofrecer calidad, pues trae consigo el realizar disminuciones importantes en los costos y gastos, diseñar procesos acordes con las necesidades del mercado y estar en permanente innovación y desarrollo, ofreciéndole al cliente lo que demanda en cada momento. Sin embargo, la supervivencia y crecimiento de las organizaciones de negocios, en un ambiente competitivo y convulsionado, dependerá mucho más de la diferenciación tecnológica, mediante el desarrollo y la coordinación de redes dinámicas de empresas.

Otra variable que se encuentra en la periferia es el proceso de control, entendida como la comparación, seguimiento y la evaluación de lo planeado versus lo ejecutado, de forma que, al encontrar hallazgos o no conformidades, se retroalimente a los procesos y se busque la mejora continua, para garantizar que los objetivos de la empresa y los planes diseñados para alcanzarlos se logren. Lo anterior se relaciona estrechamente con la función de planear; sin embargo, planear y controlar son los pilares del presupuesto, herramienta por excelencia del control que permite visualizar el desarrollo y alcance de cada uno de los indicadores de gestión diseñados en procura de la visión empresarial formulada en el plan de desarrollo.

En este sentido, el control organizacional se asimila al marco administrativo que regula las acciones de la organización para encaminarla al cumplimiento de sus propósitos. Así, el control tiene la obligación de mantener la coherencia y la consistencia de las acciones organizacionales 
en relación con sus proyectos. Por coherencia, se entiende la interrelación y conexión entre los elementos o acciones administrativas y por consistencia, el equilibrio y estabilidad del sistema (Arango, 2007). En los análisis realizados, el mercadeo es la variable más alejada de la periferia, dado que es poco frecuente encontrar estudios de tipo evaluativo del componente de mercadeo. Sin embargo, los administradores que adoptan una orientación al mercado reconocen que el marketing es vital para el éxito de sus organizaciones, lo cual se refleja en un planteamiento fundamental de los negocios que le da al cliente la máxima prioridad (Stenton, Etzel y Walker, 2007).

Para Kotler y Keller (2006), que son de los autores más representativos en términos de la gestión de marketing, en las empresas se establece que el éxito financiero suele depender del talento comercial y del marketing de las empresas. Adicionalmente, los departamentos de finanzas, producción, contabilidad o cualquier otro no importarían verdaderamente si no hubiera una demanda suficiente de los productos y servicios de la empresa que le permita obtener beneficios. Por lo anterior, es importante plantear estudios que analicen este aspecto.

Según Calderon y Castaño (2005), uno de los aspectos de la gestión de mercadeo de los empresarios PYME más destacable es su racionalidad de trabajo general a partir de una actitud pasiva que se caracteriza por esperar que los clientes hagan sus pedidos y no en una actitud proactiva de buscar nuevos mercados. Carecen de un plan de mercadeo formal, a corto, mediano y largo plazo. También afirma que una de las decisiones que más se les dificulta a los empresarios es definir a que mercados dirigirse y con qué productos específicos hacerlo. Se observa la tendencia de producir una alta gama de productos para diferentes mercados sin tener información sobre cuáles son los realmente rentables para la empresa.

Por lo anterior, el mercadeo es una disci- plina con una gran carga estratégica, científica y metodológica, una poderosa herramienta para mejorar la imagen de marca e incrementar las ventas. Realizar un buen marketing conlleva buenos resultados empresariales, siendo el marketing la clave para la competitividad empresarial, activador de la demanda y motor de la economía (De Vicuña, 2016).

Finalmente, el marketing, como herramienta de apoyo empresarial, ha adquirido gran valor en los últimos años, debido a que la adecuada ejecución y diseño de sus estrategias proporciona a las organizaciones ventajas que van desde un mejor posicionamiento estratégico, adelantándose a los movimientos de los competidores, conocer las mejores formas de distribución, precio, etc., y estar al tanto de las tendencias hacia las cuales se orientan los gustos de los consumidores. (Cepeda, Velásquez y Marín, 2017).

\section{Conclusiones}

De acuerdo con lo expuesto, se pueden hacer las siguientes consideraciones:

El núcleo de la representación social del fracaso empresarial está en el componente financiero, siendo el acceso al financiamiento la causa más incidente, considerada por los investigadores, y en una mediana incidencia la estructura de costos y el manejo de la información financiera.

Otro aspecto directamente relacionado con el núcleo es la planeación, que constituye un camino en doble vía con el acceso a la financiación; por lo que se relaciona con la variable financiera.

En un segundo plano, pero también presente en el núcleo, se encuentran el conocimiento y las competencias del recurso humano y la dimensión denominada dirección que hace parte de la gestión organizacional. 
En la periferia, con mediana incidencia, están los aspectos relacionados con la gestión organizacional y el control, al igual que la innovación y la tecnología, y el mercadeo, relacionado especialmente con la promoción y el precio.

En la periferia, y con baja incidencia, están el manejo de las relaciones interpersonales y las dimensiones de producto y plaza relacionadas con la categoría mercadeo.

De esta manera, en el núcleo de la RS se encuentran cuatro aspectos centrales: lo financiero, la gestión organizacional, la innovación y el manejo del recurso humano. Tres de ellos (Financiero, Gestión y Recurso Humano) siguen siendo los mismo que los encontrados en el estudio de Fundes (2003) La realidad de la PYME colombiana. Esto nos indica dos posibles situaciones: 1) que los investigadores han propuesto sus estudios alrededor de unos elementos que se van constituyendo en una tradición, y que, de acuerdo con la teoría de las RS, se podría considerar un sentido común instalado; $o, 2$ ), que las soluciones planteadas para el problema, las cuales, van desde la creación de líneas de financiación para las PYME a programas de formación y fortalecimiento, no han dado los resultados esperados. Es importante resaltar que las variables de la periferia, proceso de organización, el control y el marketing, aunque son importantes en la teoría de la Administración de Empresas, han sido poco abordadas, por lo que se constituyen en categorías emergentes. Estos elementos abren la posibilidad de nuevos estudios y enfoques frente al problema.

\section{Referencias bibliográfica}

ANIF. (2016). Crecimiento PYME. Coyuntura PYME.

Arango,M.R.(2007).Controlorganizacional:¿sistema o agregado de elementos? AD-MINISTER, 80-96.
Arbic, J. C. (2001). Prácticas Sociales y representaciones. México: Ediciones Coyoacán.

Beltran, A. (2006). Los 20 problemas de la Pequeña y Mediana Empresa. Dialnet, 8-15.

Berumen, S. (2008). Cambio Tecnológico e Innovación en las Empresas. Madrid: ESIC.

Calderon, G., y Castaño, G. A. (2005). Investigación en Administración en América Latina: Evolución y Resultados. Manizalez: Edigráficas.

Cámara de Comercio de Bogotá. Dirección de Gestión del Conocimiento. (2019), Causas de liquidación de las empresas 2018, https://bibliotecadigital.ccb.org.co/handle/11520/22862

Castaño, A. (2011). Planeación estratégica en la PYME de Bogotá, Orientación al futuro y evasión de la incertidumbre. Contaduría y administración, 147-173.

Cepeda, S., Velásquez, L., y Marín, B. (2017). Análisis evaluativo a los procesos de marketing en la internacionalización de las pequeñas y medianas empresas de alimentos de medellín. Estudios Gerenciales, 271-280.

Chaparro, C. I. (2012). Proceso de planeación estratégica aplicado en el contexto de la empresa boyacense. Revista In Vestigium Ire., 7-16.

Chiavenato, I. (2009). Gestión del Talento Humano. México: McGraw Hill.

Chorro, M. (2010). Financiamiento del Desarrollo. Santiago de Chile: Naciones Unidas CEPAL

Confecámaras. (2016). Informe primer trimestre. Bogotá. 
DANE. (2016a). Boletin tecnico -PIB Bogotá D.C. . Bogotá

DANE. (2016b). Cuentas Nacionales Departamentales - PIB departamental 2015 preliminar. Bogotá: Documentado informe Balance de la economía de la región Bogotá Cundinamarca 2016. Cámara de Comercio de Bogotá.

De Vicuña, A. (2016). El plan de Marketing en la PYME. Esic Editorial.

Dichiara, R. (2011). Sistemas de Organización Empresarial en Red. EAE.

Florez, A. T. (2009). La comunicación organizacional interna: Una estrategia para adaptar representaciones sociales en la organización. Filo de Palabra, 17-33.

Fundación para el Desarrollo Sostenible de las Pequeñas y Medianas empresas en América Latina FUNDES. (2003). Realidad de la PYME en Colombiana.Desafio para el desarrollo. Recuperado de: http://www.urosario.edu.co/Universidad-Ciencia-Desarrollo/ur/Fasciculos-Anteriores/Tomo-II-2007/ Fasciculo-15/pdf/2007_fa15_La_realidad_ de_la_PYME_colombiana/.

Jiménez, Absalón. (2012). Imaginario y memoria religiosa en Bogotá. Revista de antropología experimental No. 12, Texto 14, pág 175-192. Universidad de Jaén. Jaén.

Kotler, P., y Keller, K. (2006). Dirección de Marketing. México: Pearson.

Luna, A. (2014). Proceso Administrativo. México: Grupo Editorial Patria.
Méndez, C. (2009). Tecnologias y herramientas de gestión. Caso: Grandes, mediana y pequeñas empresas en Bogota. Bogotá: Universidad del Rosario.

Murdock, G. (1945). The common denominator of cultures. Nueva York: The science of man in the world crisis.

Páramo, D. (2000). La etnografía, una aproximación antropológica al comportamiento del consumidor. Revista colombiana de marketing Vol 1 No. 1. Recuperado de http://revistas.unab.edu.co/index.php?jour nal $=$ marketing\&page $=$ article\&op $=$ viewArti cle\&path\%5B\%5D=787.

Pardo, C. I., y Alfonso, W. (2015). Instituto del Fracaso. Obtenido de https://thefailureinstitute.com/wp-content/uploads/2015/11/ Fracaso-emprendimiento-Colobia.pdf

Porter, M. E., Kramer, M. R., y Lorsch, J. W. (2009). Ser competitivo (No. 338.6048 P69Y 2008.). Barcelona, España: Deusto.

Puyana, D.G. (2011) La problemática de las PYME en Colombia: intenacionalizarse o morir. https://es.scribd.com/document/59958001/ La-Problematica-De-Las-PYME-en-Colombia

Ricoeur, P. (2000). Del texto a la acción. Ensayos de hermeneùtica II. Fondo de Cultura Económica Argentina. Buenos Aires.

Rodríguez, T.; García, M.; y Jodelet, D. (2007). Representaciones Sociales: Teoría e Investigación. Guadalajara: Editorial CUCSHUdeG- 328 páginas: https://books.google. com.co/books?id=X3Eb7sKFaWMC\&print $\mathrm{sec}=$ frontcover\&source $=$ gbs_ge_summary $\mathrm{r} \& \mathrm{cad}=0 \# \mathrm{v}=$ onepage $\& \mathrm{q} \& \mathrm{f}=$ false . 
Romero, F. (2013). Variables financieras determinantes del fracaso empresarial para la pequeña y mediana empresa en Colombia: análisis bajo modelo Logit1. Pensamiento y Gestión, 235-277. Recuperado de http://www.redalyc.org/articulo.oa?id=64628626002

Romero, F.; Melgarejo, Z.A. y Vera, M.A. (Febrero de 2015). Fracaso Empresarial de las pequeñas y medianas empresas (PYME) en Colombia. Suma de negocios. Volumen 6. No. 13. Pág 29-41. Recuperado de https:// www.sciencedirect.com/science/article/pii/ S2215910X15000087

Serna, A.; Oviedo, A. y Bravo, L.F. (2010). Creencia, sistema de creencia y poder simbólico. Un propuesta para indagar los sistemas ideacionales desde la Investigación Social Interdisciplinaria en Desafíos en Estudios Sociales e Interdisciplinariedad. Bogotá: Ediciones Antropos Ltda.

Stenton, W., Etzel, M., y Walker, B. (2007). Fundamentos de Marketing. México: McGraw Hill.
Suarez, R. P., De la Rosa, L., Jimenes, B., \& Toyos, A. (2009). El Desafío de la Innovación. Cuba: Universitaria.

Vargas Hernández, J. G. (2007). La culturocracia organizacional en México. México: Juan Carlos Martínez Coll.

Vásquez Blomer, B.; Escudero Serrano, M. J., y Gabín, M. D. (2010). Empresa y Adminisstración. España: Ediciones Paraninfo S.A.

Vecina, C. (2009). Representaciones sociales: inmigración y prensa. Análisis cualitativo del discurso en los medios de comunicación. Palma de Mallorca: Ediciones Palma de Mallorca.

Villaseca Motales, D. (2014). Innovación y Marketiong de Servicios en la Era Digital. Madrid: ESIC.

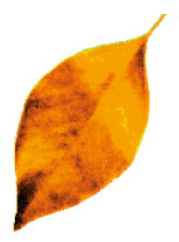

